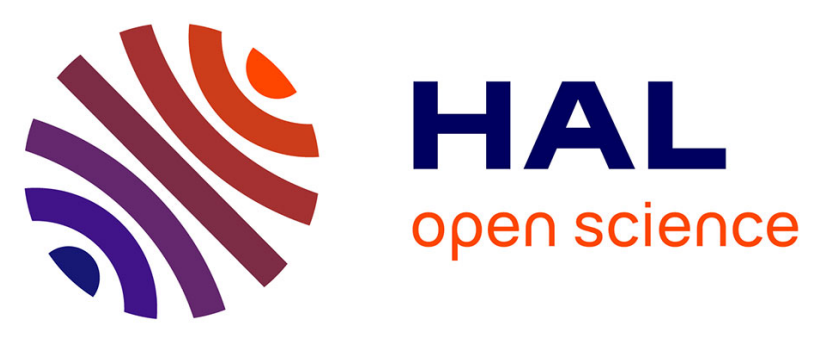

\title{
From monomers to agglomerates: A generalized model for characterizing the morphology of fractal-like clusters
}

\author{
Jerôme Yon, J. Morán, F.-X. Ouf, M. Mazur, J.B. Mitchell
}

\section{To cite this version:}

Jerôme Yon, J. Morán, F.-X. Ouf, M. Mazur, J.B. Mitchell. From monomers to agglomerates: A generalized model for characterizing the morphology of fractal-like clusters. Journal of Aerosol Science, 2021, 151, pp.105628. 10.1016/j.jaerosci.2020.105628 . hal-02923104

HAL Id: hal-02923104

https://hal-normandie-univ.archives-ouvertes.fr/hal-02923104

Submitted on 2 Sep 2020

HAL is a multi-disciplinary open access archive for the deposit and dissemination of scientific research documents, whether they are published or not. The documents may come from teaching and research institutions in France or abroad, or from public or private research centers.
L'archive ouverte pluridisciplinaire HAL, est destinée au dépôt et à la diffusion de documents scientifiques de niveau recherche, publiés ou non, émanant des établissements d'enseignement et de recherche français ou étrangers, des laboratoires publics ou privés.

\section{(1) (1) $\$$}

Distributed under a Creative Commons Attribution - NonCommercial - NoDerivatives 44.0 


\title{
From monomers to agglomerates: A generalized model for characterizing the morphology of fractal-like clusters
}

\author{
J. Yon ${ }^{\mathrm{a}, *}$, J. Morán ${ }^{\mathrm{a}}$, F.-X. Ouf ${ }^{\mathrm{b}}$, M. Mazur ${ }^{\mathrm{a}}$, J. B. Mitchell ${ }^{\mathrm{c}}$ \\ ${ }^{a}$ Normandie Univ, UNIROUEN, INSA Rouen, CNRS, CORIA, 76000 Rouen, France. \\ ${ }^{b}$ Institut de Radioprotection et de Sûreté Nucléaire (IRSN), PSN-RES, SCA, \\ Gif-Sur-Yvette, 91192, France. \\ 'SAS MERL-CONSULTING, 21 Rue Sergent Guihard, Rennes, 35000, France.
}

\begin{abstract}
The morphological description of fractal agglomerates is generally reduced to only two parameters, namely the mass fractal dimension and its prefactor. In the most evolved approaches, a stretching exponent is also introduced, while a packing factor is preferred to the fractal prefactor. In any case, the current analytical description of agglomerates morphology is accurate only for sufficiently large agglomerates, which is due to the limited spatial extension of the clusters that are actually "quasi-fractal". In the present study, a cutoff function of the pair correlation function is considered for both larger and smaller scales. This enables a more accurate morphological description valid for any cluster size is to be given taking into account the polydispersity of the primary spheres. This new analytical morphological description relying on 5 parameters, is presented here for the first time. The physical range covered by these morphological parameters is determined based on virtually generated Diffusion Limited Cluster Agglomeration. Finally, the model is used to express the fractal prefactor and structure factors and their dependence on agglomerates size and morphological parameters is investigated.
\end{abstract}

Keywords: Fractal Dimension; fractal prefactor; soot; SAXS

${ }^{*}$ Corresponding author.E-mail address: yon@coria.fr

Preprint submitted to Journal of Aerosol Science

August 31, 2020 


\section{Nomenclature}

$\beta, \beta_{0}, \beta_{\infty}$ Stretching exponent, at small and large scales. [-]

$\Gamma \quad$ The gamma function.

$\Lambda \quad$ An integral. $[-]$

$\lambda$ Wavelength. $[m]$

$\overrightarrow{d_{i j}} \quad$ Distance between centers of spheres i and j. $[\mathrm{m}]$

$\vec{q} \quad$ Scattering wave vector. $\left[m^{-1}\right]$

$\vec{r}, \vec{u}$ Position vectors. $[m]$

$\theta \quad$ Scattering angle. $[\mathrm{rad}]$

$\varphi \quad$ Packing factor. $[-]$

$\xi, \xi_{0}, \xi_{\infty}$ Equivalent spatial extension length scale, small and large scales size parameters. $[m]$

a Characteristic primary sphere radius. $[m]$

$A, A_{p p}, A_{a g g}$ Pair correlation function, for primary sphere self-intersection, for different primary spheres intersection. $\left[\mathrm{m}^{3}\right]$

$C \quad$ Constant of proportionality. $\left[m^{6-D_{f}}\right]$

$D_{f}, D_{f i}, D_{f p}$ Particle fractal dimension, individual particle and population based dimension. $[-]$

$f_{p p} \quad$ The probability density function of the primary spheres. $\left[\mathrm{m}^{-1}\right]$

$k_{f}, k_{f i}, k_{f i, \infty}, k_{f p}$ Particle's fractal prefactor, individual one, individual one for infinitely large agglomerates and population based one. [-3]

$n(\vec{r})$ Indicator of presence of material. $[-]$

$N_{p} \quad$ Number of primary spheres per agglomerate. [-] 
$r \quad$ Radial distance. $[m]$

$R_{g} \quad$ Radius of gyration. $[m]$

$R_{p}, R_{p i}, R_{p v}$ Primary sphere radius, for the $i$ th sphere, volume equivalent primary sphere. $[m]$

$S, S_{\infty}$ Structure factor, structure factor for infinitely large agglomerates. [-]

$V, V_{p}, V_{a}$ Volume of intersection between two spheres, volume of a primary sphere and of the agglomerate. $\left[\mathrm{m}^{3}\right]$ 


\section{Highlights}

- An analytical form of the pair correlation function is proposed.

- Morphological description independent of agglomerates size.

- Fractal prefactor and structure factor as a function of size.

\section{Graphical Abstract}

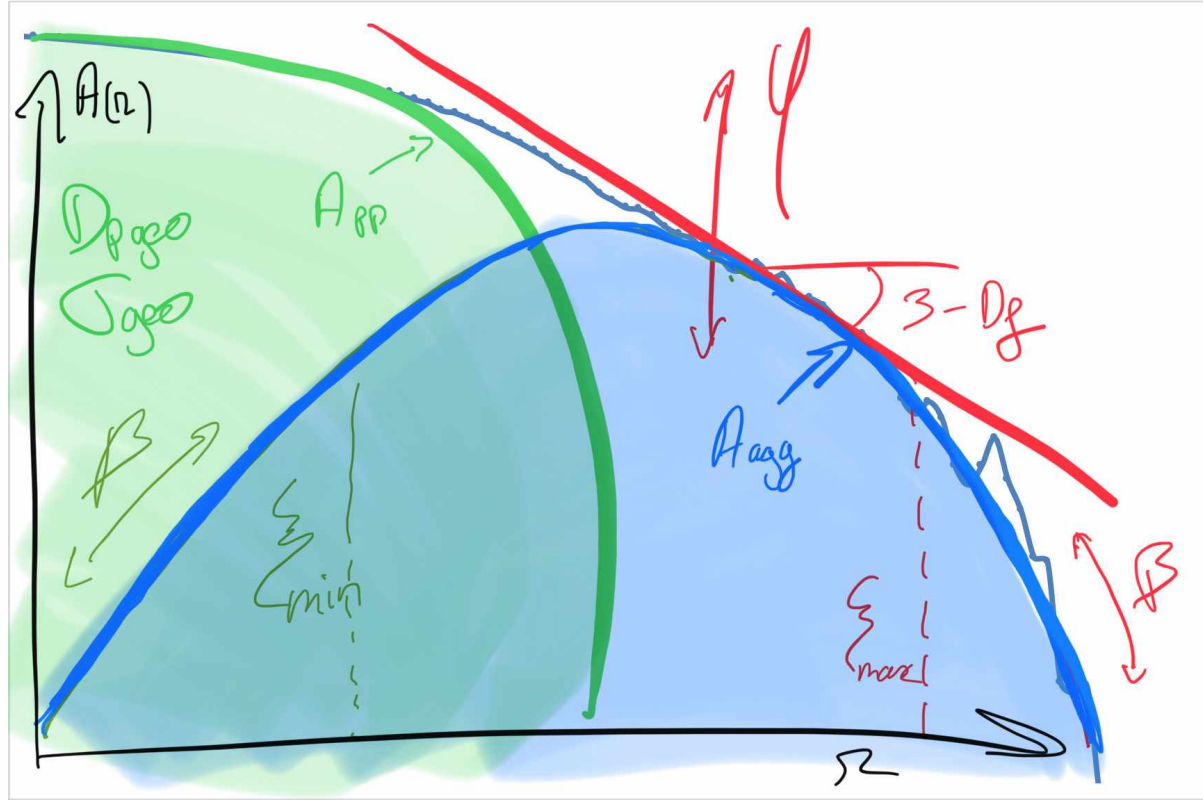




\section{Introduction}

Agglomeration is a phenomenon observed for both aerosols and colloids and of particular importance in nanoscience. Agglomerates thus generated often exhibit a ramified structure due to the random nature of their Brownian movement. In many cases, an empirical power-law relationship between the agglomerate mass and size is observed such as the very commonly used fractal law Meakin (1991); Jullien (1992); Bushell et al. (2002). When this is plotted in a normalised log-log graph, the slope and the intercept are related to the population based mass fractal dimension $D_{f p}$ and prefactor $k_{f p}$ respectively:

$$
N_{p}=k_{f p}\left(\frac{R_{g}}{a}\right)^{D_{f p}}
$$

where $N_{p}$ is the number of primary spheres, $R_{g}$ the radius of gyration of a given agglomerate and $a$ is a characteristic primary sphere radius. This equation is very useful in many applications and extremely popular due to its simplicity. It is well accepted that the mass fractal dimension is related to the agglomeration regime (typically $D_{f p} \simeq 1.78$ in diffusion limited regimes and up to 1.91 in ballistic agglomeration) Meakin (1999), with a high value for more "compact" particles. Much less attention has been paid to $k_{f p}$, that is shown to be related to a greater dispersion $\left(k_{f p} \simeq 1\right.$ in simulations and up to 5 in experimental observations Wu and Friedlander (1993); Lapuerta et al. (2010)). Moreover, its physical meaning is less clear, even if different studies have been conducted with the aim of exhibiting a relationship between $k_{f p}$ and $D_{f p}$ Sorensen and Roberts (1997); Ehrl et al. (2009) or between $k_{f p}$ and $N_{p}$ Lapuerta et al. (2010); Gmachowski (2002). Some authors Ehrl et al. (2009); Lazzari et al. (2016); Isella and Drossinos (2010); Lattuada et al. (2003) have shown that an agglomerate needs to be constituted of at least 15 to 30 primary spheres before $k_{f p}$ arrives at an asymptotic behaviour, which highlights the limited validity of the fractal law Eq. 1 for smaller clusters. Still, in many cases, the fractal law is used for clusters with a limited number of primary spheres Köylü et al. (1997); Lee et al. (2000); Wentzel et al. (2003); Cortés et al. (2018). For example, for soot particles, most of the particles contain nearly 60 primary spheres in stationary diffusion flames Kholghy et al. (2013) or even less for shorter flame residence time Cortés et al. (2018); Bladh et al. (2011); Gigone et al. (2019); Altenhoff et al. (2019). For such applications, the question arises on the validity of the use of the fractal law for Scanning/Transmission Electron Microscopy 
(SEM/TEM) analysis Wozniak et al. (2012) as well as for mobility diameter interpretation Betrancourt et al. (2017); Tang et al. (2017) or X-ray or visible light scattering diffraction pattern analysis Yon et al. (2018); Bouvier et al. (2019); Sorensen et al. (1995). For instance it is not known, whether the size dependence of the fractal prefactor needs to be considered, and whether one should extend current light-agglomerate interaction models such as the Rayleigh Debye Gans theory for Fractal Agglomerates (RDG-FA) in the visible domain Sorensen (2001) or the Beaucage analysis for Small Angle X-ray (SAXS) analysis Beaucage (1995) for quasi-fractal agglomerates. In order to answer these questions, it is necessary to characterize the morphology of individual agglomerates, and the most suitable tool is probably the pair correlation function:

$$
A(\vec{r})=\int_{\vec{u}=\vec{o}}^{\infty} n(\vec{r}-\vec{u}) n(\vec{u}) d \vec{u}
$$

where $n(\vec{r})$ is 1 if $\vec{r}$ points towards the material and 0 otherwise. When $A$ is averaged over the agglomerate orientations, the result becomes a smooth function of $r$. It can be demonstrated that the agglomerate volume $V_{a}$ and the radius of gyration $R_{g}$ can be determined based on a knowledge of $A(r)$ (see Appendix of Sorensen (2001) and ref. Nicolai et al. (1994)):

$$
\begin{aligned}
V_{a}^{2} & =\int 4 \pi r^{2} A(r) d r \\
R_{g}^{2} & =\frac{1}{2} \frac{\int_{r=0}^{\infty} r^{4} A(r) d r}{\int_{r=0}^{\infty} r^{2} A(r) d r}
\end{aligned}
$$

In the case of an ideal fractal cluster, the average pair correlation function should exhibit a power-law behaviour $\left(A(r) \propto r^{D_{f i}-3}\right)$ whose slope in a $\log -\log$ plot is related to the cluster individual fractal dimension $D_{f i}$. Theoretically, the average of $D_{f i}$ over the entire population of particles should be $D_{f p}$. However, real agglomerates have a finite size and a cutoff function needs to be added Sorensen (2001); Cai et al. (1995); Rottereau et al. (2004); Lin et al. (1990); Sorensen et al. (1992a):

$$
A(r) \propto r^{D_{f i}-3} \exp \left(-(r / \xi)^{\beta}\right)
$$

The cutoff is driven by a spatial extension $\xi$ and a stretching exponent $\beta$ Nicolai et al. (1994). The latter parameter plays an important role on the scattering properties Sorensen (2001) and, as demonstrated by Heinson et al. (2012), 
is correlated with the particle anisotropy. The same authors have introduced a packing factor $\varphi$, which expresses the local arrangement of neighbouring spheres and ensures mass conservation (respect of Eq. 3):

$$
A(r)=\frac{\varphi D_{f i}}{4 \pi R_{p}^{D_{f i}}} r^{D_{f i}-3} \exp \left(-(r / \xi)^{\beta}\right)
$$

with $R_{p}$ the monomer primary sphere. By applying this last expression for the pair correlation function in Eq. 4 and the corresponding expression for the radius of gyration into Eq. 1, Heinson et al. (2012) have shown the link between the fractal prefactor and the three parameters $D_{f i}, \varphi$ and $\beta$ for large agglomerates,

$$
k_{f i, \infty}=\left[\frac{2 \Gamma\left(D_{f i} / \beta\right)}{\Gamma\left(\left(D_{f i}+2\right) / \beta\right)}\right]^{\frac{D_{f i}}{2}} \frac{\varphi D_{f i} \Gamma\left(D_{f i} / \beta\right)}{\beta}
$$

The authors introduce an individual prefactor $k_{f i}$ based on the underlying assumption that the entire population of an agglomerate has identical $\varphi$, $D_{f i}=D_{f p}$ and $\beta$ parameters. Equation 7 highlights the complexity of the prefactor due to its dependence on these three parameters, which partly explains the dispersity of the reported values of $k_{f p}$ in the literature even without considering primary sphere overlapping Brasil et al. (1999). To the authors' knowledge, until now the work reported by Heinson et al. (2012) is the most up to date and relevant on this subject. However, it is only valid for agglomerates consisting of monodisperse monomers whose number is large enough to ensure the validity of Eq.6 i.e. neglecting smaller scales. Therefore, Eq.7 should not be considered for clusters for which $N_{p}$ is less than approximately 30 .

In addition to the morphological signature embedded in the pair correlation function, one particularly interesting feature of this function is that its normalized Fourier transform corresponds to the particle structure factor, i.e. the description of its light scattering pattern Debye et al. (1957):

$$
S(\vec{q})=\frac{\int A(\vec{r}) \exp (i \vec{q} \cdot \vec{r}) d \vec{r}}{\int A(\vec{r}) d \vec{r}}
$$

where $\vec{q}$ is the scattering wave vector whose norm is $q=\frac{4 \pi}{\lambda} \sin (\theta / 2)$. By definition, the structure factor is 1 in the case of forward scattering $(\theta=0)$. $S(q)$ is thus a modulating factor for the forward light scattering intensity 
enabling a description to be given of the impact of the light collection angle $\theta$ Oltmann et al. (2010); Kempema and Long (2016); Bushell and Amal (2000) and wavelength $\lambda$ Lefevre et al. (2019); Bouvier et al. (2019) on the light scattering signal. The main assumption is that each constituent of the particle individually scatters the incident light without any disturbance by other components. This is valid in the Rayleigh scattering regime (particles small compared to the wavelength) and if the refractive index is close to 1 (X ray scattering, cf SAXS, neutron scattering, etc.) Zhao et al. (2007). The modelling of the structure factor thus provides a quantitative interpretation of light scattering patterns in terms of particle size and shape. For agglomerates, this function has been used to provide the most simple analytical expressions of the structure factor possible. While the community concerned by visible light often employs the RDG-FA, see Refs. Sorensen (2001)), the community working with $\mathrm{X}$ rays or short wavelengths as in SAXS, more usually uses the Beaucage model Beaucage (1995); Beaucage et al. (2004). In any case, those two models also consider clusters large enough to express their fractal signature.

For these reasons, Lattuada et al. (2003) focused on the pair correlation of agglomerates with a number of primary spheres below 100 . They observed a transition regime at smaller scales that was empirically modeled by a new power law regime. However, the primary sphere volume was not considered in their numerical determination of the pair correlation function and the parameters of their model do not allow a physical interpretation of the phenomena involved to be made. Therefore, the purpose of the present study is to pursue that investigation by improving the numerical computation of the pair correlation and its modelling by considering the primary sphere volume and a new cutoff function for smaller scales. Another aim is to consider the effect of primary sphere polydispersity. Moreover, the study will show that a finer description of the pair correlation function at both small and large scales enables the validity of the fractal law and structure factor to be extended to all agglomerate sizes.

\section{Proposed model}

\subsection{Pair correlation}

As explained in introduction, the validity of the fractal law and of the structure factor for smaller particles is limited by the fact that the pair correlation function behaviour at small scales is not taken into account, most 
certainly due to the current numerical way of computing $A(r)$ (discussed later in section 3.1. In fact, the modelling of the pair correlation function can be decomposed into two distinct terms $A_{p p}$ and $A_{a g g}$ :

$$
A(\vec{r})=\underbrace{\sum_{i=1}^{N_{p}} V\left(R_{p i}, R_{p i}, \vec{r}\right)}_{A_{p p}}+\underbrace{\sum_{i=1}^{N_{p}} \sum_{j=1 \& j \neq i}^{N_{p}} V\left(R_{p i}, R_{p j}, \overrightarrow{d_{i j}}\right)}_{A_{a g g}}
$$

where $V\left(R_{p i}, R_{p j}, \overrightarrow{d_{i j}}\right)$ represents the interception volume between two spheres of radius $R_{p i}$ and $R_{p j}$ respectively, for which the distance between respective centers is $d_{i j}$. The first term $A_{p p}$ represents the contribution of the intersection of spheres with themselves whereas $A_{\text {agg }}$ represents the interception between different spheres. For agglomerates made of point contact spheres, $A_{p p}$ can be simplified to:

$$
A_{p p}(r)=\sum_{i=1}^{N_{p}} V_{p i}\left(1+\frac{r}{4 R_{p i}}\right)\left(1-\frac{r}{2 R_{p i}}\right)^{2}, \quad r \in\left[0,2 R_{p i}\right]
$$

where $V_{p i}$ is the volume of the $i$ th monomer. Since particle self interaction $\left(A_{p p}\right)$ is now considered for short scales, a cutoff must also be considered for smaller scales in the expression for $A_{\text {agg }}$. Indeed, for just touching spheres, $A_{a g g}$ is supposed to tend towards 0 when $r \rightarrow 0$. Therefore, a cutoff function for small scales, similar to those currently used at larger scales, is added to the model:

$$
A_{a g g}(r) \propto\left(1-\exp \left(-\left(r / \xi_{0}\right)^{\beta_{0}}\right)\right) r^{D_{f i}-3} \exp \left(-\left(r / \xi_{\infty}\right)^{\beta_{\infty}}\right)
$$

In this equation, $\xi_{0}$ and $\xi_{\infty}$ refer to the smaller and larger length scales respectively and $\beta_{0}$ and $\beta_{\infty}$ to the stretching exponents relative to smaller and larger length scales respectively. In order to reduce the number of parameters, the following assumption, justified later, will be used: $\beta_{0}=\beta_{\infty}=\beta$. This hypothesis enables a simplification of Eq. 11 to be made, which becomes:

$$
A_{\text {agg }}(r)=C r^{D_{f i}-3}\left[\exp \left(-\left(r / \xi_{\infty}\right)^{\beta}\right)-\exp \left(-(r / \xi)^{\beta}\right)\right]
$$

58 where $\xi=\left[\xi_{0}^{-\beta}+\xi_{\infty}^{-\beta}\right]^{-1 / \beta}$ and $C$ is a proportionality constant. This form 59 is of interest for the following analytical developments. 


\subsection{Volume and radius of gyration}

Based on Eqs. 3, 10 and 12, the agglomerate's volume $V_{a}$ has to respect the following equation:

$$
V_{a}=\frac{\overline{V_{p}^{2}}}{\overline{V_{p}}}+\frac{4 \pi C}{\beta V_{a}} \Gamma\left(\frac{D_{f i}}{\beta}\right)\left(\xi_{\infty}^{D_{f i}}-\xi^{D_{f i}}\right)
$$

\subsection{Fractal prefactor}

Knowing that, for point contact spheres, $N_{p}=\frac{V_{a}}{V_{p}}$, Eq. 15 can be used to express an individual prefactor based on Eq. 1. In the present study, the characteristic radius $a$ is arbitrarily considered equal to the volume equivalent primary sphere radius $R_{p v}=\left(\frac{3 \overline{V_{p}}}{4 \pi}\right)^{1 / 3}$ :

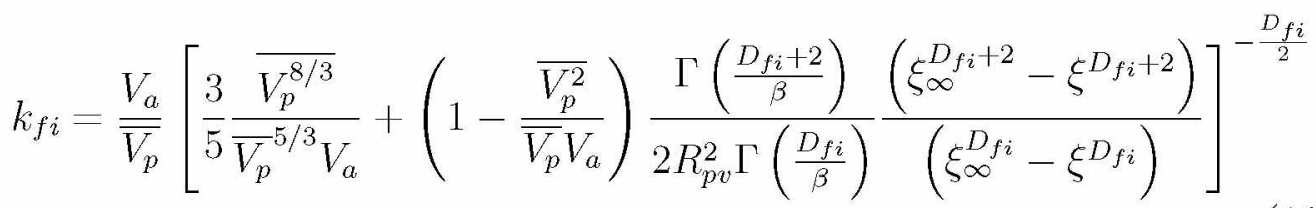




\subsection{Asymptotic cases $N_{p}=1$ and infinitely large agglomerates}

For an isolated sphere, the second term in brackets in Eq. 16 becomes zero reducing the equation to the well known expression $k_{f i, p p}=(3 / 5)^{-\frac{D_{f i}}{2}}$. For infinitely large clusters, the first term in brackets in Eq. 16 becomes negligible as well as $\xi^{D_{f i}+2}$ and $\xi^{D_{f i}}$ compared to $\xi_{\infty}^{D_{f i}+2}$ and $\xi_{\infty}^{D_{f i}}$ respectively. The same kind of simplification can be done with Eq. 13 for infinitely large clusters. By combining both simplified expressions for $k_{f i}$ and $V_{a}$ respectively, the analytical expression for the asymptotic individual prefactor becomes:

$$
k_{f i, \infty}=\left[\frac{2 \Gamma\left(D_{f i} / \beta\right)}{\Gamma\left(\left(D_{f i}+2\right) / \beta\right)}\right]^{\frac{D_{f i}}{2}} \frac{3 R_{p v}^{D_{f i}-3} C \Gamma\left(D_{f i} / \beta\right)}{\beta V_{a}}
$$

This expression is similar to the result found by Heinson et al. (2012) recalled in Eq. 7. The comparison allows the constant $\mathrm{C}$ to be expressed as a function of the packing factor:

$$
\frac{C}{V_{a}}=\frac{\varphi D_{f i}}{3 R_{p v}^{D_{f i}-3}}
$$

These results show that the generalized $k_{f i}$ (Eq. 16) displays correct asymptotic behaviours. The constant $C$ is proportional to the agglomerate volume but modulated by the packing factor, the primary sphere radius and the polydispersity. Finally, the same simplifications that are valid for infinitely large agglomerates can be made for the expression for the radius of gyration (Eq. 15), which leads to the following relation:

$$
\frac{\xi_{\infty}}{R_{g}}=\left[\frac{2 \Gamma\left(\frac{D_{f i}}{\beta}\right)}{\Gamma\left(\frac{D_{f i}+2}{\beta}\right)}\right]^{1 / 2}
$$

This relation has already been proposed by Nicolai et al. (1994) in 1994. This ratio $\xi_{\infty} / R_{g}$, is in agreement with the ratio of the cluster's maximum projected length and its gyration diameter determined numerically by Köylü et al. (1995) and Brasil et al. (1999). Thus, for large clusters, $2 \xi_{\infty}$ can be seen as representative of the agglomerate's maximum projected length.

\subsection{Structure factor}

The newly developed model for the pair correlation function (Eq. 9, 10, 12) can now be incorporated into equation 8 in order to determine an analytical 
form of

$$
\begin{aligned}
S(q)= & \frac{1}{N_{p}{\overline{R_{p}^{3}}}^{2}} \int_{0}^{\infty} 9 R_{p}^{6} \frac{\left[\sin \left(q R_{p}\right)-q R_{p} \cos \left(q R_{p}\right)\right]^{2}}{\left(q R_{p}\right)^{6}} f_{p p}\left(R_{p}\right) d R_{p} \\
& +\left(1-\frac{1}{N_{p}} \frac{\left.{\overline{R_{p}^{6}}}_{{\overline{R_{p}^{3}}}^{2}}^{2}\right) \frac{\beta}{q^{D} f i \Gamma\left(\frac{D_{f i}}{\beta}\right)} \frac{\Lambda\left(q, \xi_{\infty}, D_{f i}, \beta\right)-\Lambda\left(q, \xi, D_{f i}, \beta\right)}{\xi_{\infty}^{D_{f i}}-\xi^{D} f i}}{}\right.
\end{aligned}
$$
$\Lambda\left(q, \xi, D_{f i}, \beta\right)=\int_{0}^{\infty}(q r)^{D_{f i}-1} e^{-\left(\frac{r}{\xi}\right)^{\beta}} \frac{\sin (q r)}{q r} d(q r)$.

For isolated primary spheres, the second term of Eq. 20 vanishes and the first term simplifies to the well known expression for the structure factor for a distribution of spherical particles. At large $q$, this relation presents oscillations whose envelope has a slope of -4 when represented in a log-log plot (Porod's regime). One the other hand, for an infinitely large agglomerate, the first term becomes negligible as well as $\Lambda\left(q, \xi, D_{f i}, \beta\right)$ compared to $\Lambda\left(q, \xi_{\infty}, D_{f i}, \beta\right)$ and $\xi^{D_{f i}}$ compared to $\xi_{\infty}^{D_{f i}}$. Finally, by replacing $\xi_{\infty}$ by its expression as a function of $R_{g}(19)$, one obtains:

$$
S_{\infty}(q)=\underbrace{\frac{\beta \Lambda\left(q, \xi_{\infty}, D_{f i}, \beta\right)}{\Gamma\left(\frac{D_{f i}}{\beta}\right)}\left[\frac{\Gamma\left(\frac{D_{f i}+2}{\beta}\right)}{2 \Gamma\left(\frac{D_{f i}}{\beta}\right)}\right]^{D_{f i} / 2}}_{B\left(q, \xi_{\infty}, D_{f i}, \beta\right)}\left(q R_{g}\right)^{-D_{f i}}
$$

This corresponds to the asymptotic form of the structure factor in the powerlaw regime. $\Lambda$ has an analytical expression in the particular case of infinitely large particles and for $0<D_{f i}<2$. This enables the expression of $B$ to be written as:

$$
B\left(q, \xi_{\infty} \rightarrow \infty, D_{f i}, \beta\right)=\sin \left(\frac{\pi}{2}\left(D_{f i}-1\right)\right) \frac{\beta \Gamma\left(D_{f i}-1\right)}{\Gamma\left(\frac{D_{f i}}{\beta}\right)}\left[\frac{\Gamma\left(\frac{D_{f i}+2}{\beta}\right)}{2 \Gamma\left(\frac{D_{f i}}{\beta}\right)}\right]^{D_{f i} / 2}
$$

This expression has been previously developed by Heinson et al. (2012) and before that by Nicolai et al. (1994). In these works however, the mathematical hypothesis $0<D_{f i}<2$ was not mentioned. Nevertheless, by comparing this analytical expression with a numerical solution, it seems that expression of 
$B$ is correct for $0<D_{f i}<3$ at least if $\beta \leq 2.5$. It is interesting to observe that the power-law regime is not affected by polydispersity except if $D_{f i}$ or $\beta$ are intrinsically affected by the primary sphere polydispersity, as explained in the next section.

The novelty of the proposed approach is to provide a generalized expression for the structure factor (Eq. 20), which is valid for all numbers of primary spheres and takes into account the primary sphere polydispersity.

This advantage can be illustrated by observing the particular case of forward light scattering $(q \rightarrow 0)$ for an agglomerate consisting of monodisperse primary spheres. By applying a limited development of the functions in Eq. 20 and by using the definition of the radius of gyration, one can show that:

$$
S(q \rightarrow 0)=\frac{V_{p}}{V_{a}}+\left(1-\frac{V_{p}}{V_{a}}\right)\left(1+\frac{q^{2}}{3} \frac{R_{g}^{2}-\frac{V_{p}}{V_{a}} \frac{3}{5} R_{p}^{2}}{1-\frac{V_{p}}{V_{a}}}\right)
$$

For large agglomerates, the well known result $S(q \rightarrow 0)=1+\frac{\left(q R_{g}\right)^{2}}{3}$ related to the Guinier regime can be found but, if the number of primary spheres tends toward 1 , the structure factor at $q=0$ becomes constant and equal to 1. Equation 23 also allows the minimum number of primary spheres needed for the Guinier regime to becomes valid to be evaluated. Equation 20 can be used to answer the same question in the polydisperse case, for any scattering angle or wavelength.

\section{Results}

\subsection{Determination of the model's parameters}

In order to evaluate the parameters of the model, a statistical analysis was conducted on Diffusion Limited Cluster Agglomerates (DLCA) made up of mono- and polydisperse primary spheres. The code MCAC Morán et al. (2020); Morán et al. (2020) has been used, forcing the agglomeration to stay within the Epstein regime in order to generate standard DLCA morphologies. The volume fraction was fixed at $1 \mathrm{ppm}$, temperature $1700 \mathrm{~K}$ and $1 \mathrm{~atm}$ of pressure, the primary sphere median diameter at $D_{p, g e o}=80 \mathrm{~nm}$ and $\sigma_{p, g e o}=$ 1.45 for the case of polydisperse monomers. A total number of 27,342 and 24,150 agglomerates have been simulated for monodisperse and polydisperse monomers respectively. The classical fitting of $N_{p}$ vs $R_{g} / R_{p v}$ by the fractal law (1) enables (Fig. 6) the population fractal dimensions and prefactors to be determined respectively $D_{f p}=1.77$ and $k_{f p}=1.58$ for monodisperse and 
$D_{f p}=1.77$ and $k_{f p}=1.69$ in the polydisperse cases. Those values are typical of DLCA generated fractal agglomerates Eggersdorfer and Pratsinis (2012); Bushell and Amal (1998).

Regarding the numerical computation of the pair correlation, most of the studies in the literature only considered the position of the centers of the spheres and not their volume. This makes some peaks in $A(r)$ to appear for distances $r \leq 4 R_{p}$ Filippov et al. (2000); Lattuada and Ehrl (2009); Lattuada et al. (2003). Additionally, the application of this method is commonly limited to monodisperse point-touching monomers. A few studies have attempted to calculate it for more complex morphologies, including the partial distance distribution function introduced by Bushell and Amal (1998) and the volume-based pair correlation function introduced by Morán et al. (2019). The latter is used in the present study because it has the advantage of considering the contribution of primary particles and is not limited to the specific primary particle size distribution, shape or level of overlapping between monomers. The pair correlation function is then calculated based on 300 random orientations and 200 radial positions Morán et al. (2019).

Concerning the fitting process, for each agglomerate, the radii of the constituting primary spheres are known as well as the agglomerate volume $\left(V_{a}\right)$ and radius of gyration $\left(R_{g}\right)$. The model is thus reduced to only four unknown parameters, namely $D_{f i}, \beta, \xi_{\infty} / R_{g}, \xi_{0} / R_{p v}$. The necessity to search for $D_{f i}$ or to impose it, is discussed in the next section. The normalized pair correlation is fitted with the following expression of the model which comes from Eq. 12 after replacing $C$ by Eq.18:

$$
\begin{aligned}
& \frac{A(r)}{V_{a}}=\frac{1}{N_{p} R_{p v}^{3}} \underbrace{\sum_{i}^{N_{p}}\left(R_{p i}^{3}-\frac{3}{4} r R_{p i}^{2}+\frac{r^{3}}{16}\right)} \\
& +\frac{\varphi D_{f i}}{3}\left(\frac{r}{R_{p v}}\right)^{D_{f i}-3}\left(e^{-\left(\frac{r}{R_{g}} \frac{R_{g}}{\xi_{\infty}}\right)^{\beta}}-e^{-\left(\frac{r}{R_{p v}} R_{p v}\right)^{\beta}}\right)
\end{aligned}
$$

The parameter $\varphi$ in Eq. 24 is obtained from the agglomerate's volume by rewriting Eq. 13 which is :

$$
\varphi=\left(V_{a}-\frac{\overline{V_{p}^{2}}}{\overline{V_{p}}}\right) \frac{3 \beta}{4 \pi D_{f i}} \Gamma\left(\frac{D_{f i}}{\beta}\right)\left(\left(\frac{R_{g}}{\xi_{\infty}}\right)^{-D_{f i}}\left(\frac{R_{g}}{R_{p v}}\right)^{D_{f i}}-\left(\frac{R_{p v}}{\xi}\right)^{-D_{f i}}\right)
$$



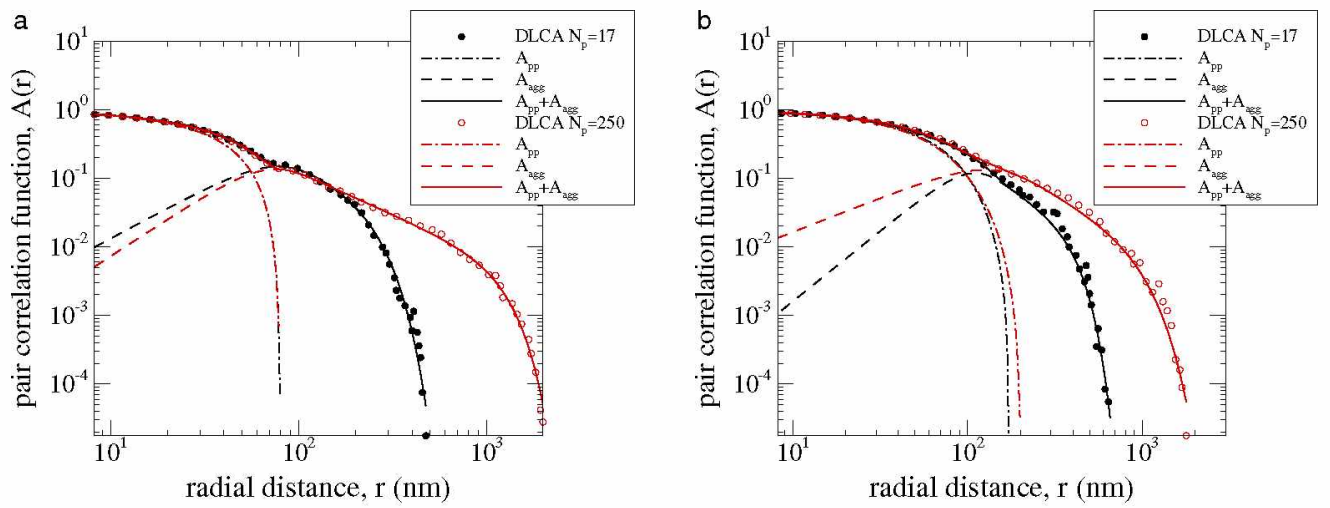

Figure 1: Examples of normalized pair correlation functions (dots), the contribution of primary particles $A_{p p}$ (dash-dotted line), the contribution of the agglomerate $A_{a g g}$ (dashed line) and the total fit (continous line). Figure (a) and (b) correspond to monodisperse and polydisperse $\left(\sigma_{p, g e o}=1.45\right)$ primary particles, respectively. Chosen agglomerates consist of $N_{p}=17$ (black color) and $N_{p}=250$ (red color).

Four examples of pair correlation functions are presented by different symbols in Fig. 1. The left half of the figure presents the fitting for two agglomerates made up of 17 and 250 monodisperse primary spheres respectively whereas the right half reports the results for polydisperse primary spheres $\left(\sigma_{p, g e o}=1.45\right)$. The fit is very good in all studied cases, showing that the assumption of a unique $\beta$ holds for a correct fitting process. The model also succeeds in taking into account, the hump present at the transition between $A_{p p}$ (dash lines) and $A_{a g g}$ (long dashed lines). It can be observed that the contribution from primary particles $A_{p p}$ is independent of $N_{p}$ for monodisperse monomers and slightly dependent of $N_{p}$ for polydisperse monomers. Also, $A_{\text {agg }}$ shows a clear difference for small and large $N_{p}$. This dependence is studied systematically in the following sections.

\subsection{Mean individual fractal dimension $D_{f i}$}

In Fig. 2, the individual monodisperse and polydisperse fractal dimensions as reported as a function of the number of primary spheres per agglomerate. A few error bars are added to represent the $95 \%$ interval confidence for illustration purposes but they are not shown everywhere to avoid cluttering the figure. Firstly, a variation of the individual fractal dimension at least up to $N_{p} \approx 30$ can be observed, even if for small agglomerates, the domain of the pair correlation function used for determining the fractal dimension is too 
small to obtain a reliable result, as indicated by the large error bars at small $N_{p}$. From this point of view, imposing $D_{f i}$ to have its asymptotic value can be considered without having significant impact on the quality of the fit. Since there is a large dispersion of $D_{f i}$ for a given number of primary spheres, the difference between fractal dimensions for the monodisperse and polydisperse cases does not appear to be significant, thus indicating that polydispersity does not seem to significantly affect the individual fractal dimension. They both have a common asymptotic value of 1.64. At first, it might appear surprising to observe a lower asymptotic individual fractal dimension than that observed in the population based fractal plot, typically equal to 1.77 , as indicated in the previous section. However, this difference between fractal dimensions determined by population and individual approaches has already been reported by Wozniak et al. (2012), who also found a lower individual fractal dimension (1.66 for Diesel soot particles) by using a Box-Counting method compared to $D_{f p}=1.88$ obtained by using the classical fractal-law. More recently Altenhoff et al. (2020) found an average asymptotic fractal dimension $\sim 1.61$ for individual agglomerate (obtained also by Box-Counting) for soot particles from premixed diffusion flames while the population one is within the $D_{f p}=1.70-1.82$ depending on the method used. An explanation for this difference is suggested in this section.

In what follows, the pair correlation model fitting is systematically performed by considering two scenarios. First, by imposing $D_{f i}$ to have its asymptotic value (dashed lines Fig. 2) and secondly, by letting the individual fractal dimension $D_{f i}$ be free. In any cases however, $D_{f i}$ is not imposed to be equal to $D_{f p}$ as previously done by Heinson et al. (2012) since it clearly appears that it does not correspond to the asymptotic behaviour exhibited by Fig. 2.

\subsection{The packing factor $\varphi$}

The packing factor is not directly determined during the fitting process but is used as a constraint (see Eq. 25). The corresponding dependence on the number of primary spheres per agglomerate (see left-hand part of Fig. 3) is found to be highly dependent on whether the fractal dimension is imposed or left free. This factor quantifies the local compactness in the vicinity of a given primary sphere whereas fractal dimension is the general, scale dependence of the compactness of the agglomerate. The imposed $D_{f i}$ case is considered in the following since it produces a monotonous increase of the packing factor. In the monodisperse case, this parameter is found to increase, 


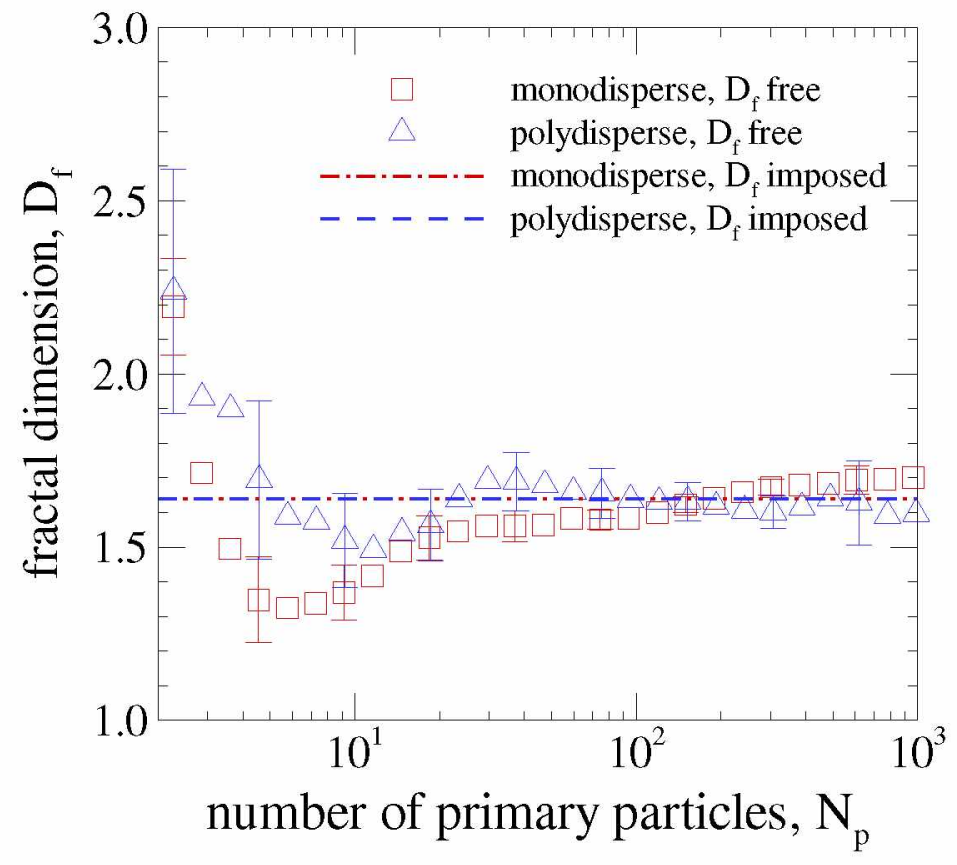

Figure 2: Fitted individual $D_{f i}$ as a function of the number of primary particles. Error bars correspond to the $95 \%$ confidence intervals.

tending towards an asymptotic value close to 1.0, which is larger than the value proposed by Heinson et al. (2012) for large DLCA agglomerates (0.68). However, it should be noted that the latter value was determined by the authors by interpreting the product $\varphi \times D_{f}$ by considering $D_{f i}=1.8$, thus explaining the lower determination of the packing factor than in the present study, as can be verified by examining Eq. 25. It should also be noted that, unlike the aforementioned work, $\varphi$ is not constant, indicating that the local compactness increases with agglomerate size. Because for point-contact cluster-cluster agglomerates the only way to increase the local compactness at the vicinity of the primary sphere is to increase the number of contacts between spheres (coordination number), the main explanation of the observed increase of the packing factor is the increase of the coordination number (see Appendix B). This is certainly due to the fact that larger structures present a larger accessible surface area for cluster collisions enabling a more important local compactness the occur when the cluster is large. The present result seems to indicate that local compactness can be further increased with 
the primary sphere polydispersity (blue triangles tending to $\varphi \approx 1.4$ ) and the number of primary spheres necessary to reach an asymptotic value seems also to be increased. The continuous curves in the imposed fractal dimension case corresponds to purely empirical fittings that will be used in section 3.6.
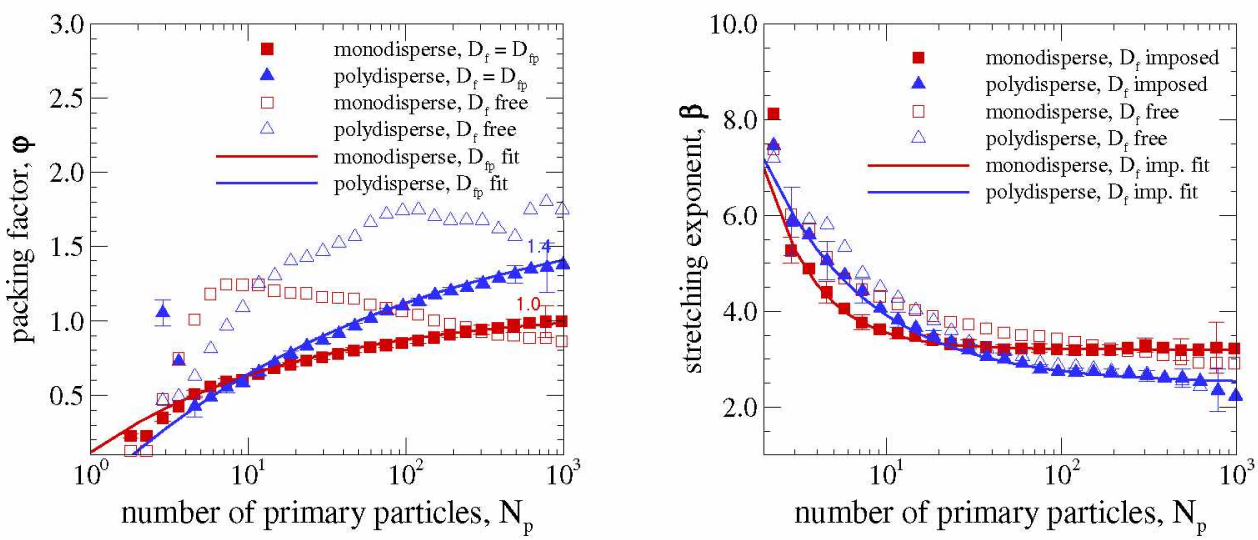

Figure 3: Fitted packing factor and stretching exponent as a function of the number of primary particles. The error bars correspond to the $95 \%$ confidence intervals.

\subsection{The stretching exponent $\beta$}

The stretching exponent is reported in the right-hand part of Fig. 3. Whether the individual fractal dimension is imposed or not, $\beta$ experiences a strong decrease with the agglomerate size increase. For small agglomerates, $\beta$ tends towards $\beta_{0}$ (small scales) whereas it tends towards $\beta_{\infty}$ for large agglomerates (domination of large scales). So, the right-hand part of Fig. 3 indicates that the probability for finding a primary sphere, which corresponds to the meaning of $A(r)$, decreases more sharply for small structures than for larger ones. This is related to the increasing number of possible configurations for positioning a new primary sphere on the outer shell of an increasingly large agglomerate. Unlike $\varphi$, an asymptotic behaviour is found for large $N_{p}$. The corresponding asymptotic value is found to be larger for monodisperse agglomerates $\left(\beta_{\infty}=3.2\right)$ than for polydisperse ones $\left(\beta_{\infty}=2.6\right)$. The polydisperse value is in better agreement with Heinson et al. (2012) than the monodisperse primary sphere case where they determined $\beta_{\infty}=2.5$. Heinson et al. (2012) have shown that $\beta$ is correlated to the particle anisotropy and fractal prefactor. Indeed, we will see that the fractal prefactor is also increased in the polydisperse case. 


\subsection{Small $\xi_{0}$ and large $\xi_{\infty}$ scales characteristic size parameters}

As explained in section 2, the agglomerate term $A_{a g g}$ of the pair correlation function is delimited by small $\xi_{0}$ and large $\xi_{\infty}$ scale size parameters. Those parameters are presented respectively in the left- and right-hand parts of Fig. 4 after being made dimensionless by the volume equivalent primary radius $R_{p v}$ and the radius of gyration $R_{g}$ respectively. The former increases with the agglomerate size, which can be explained by a numerical compensating effect due to the decrease of the $\beta$ parameter. That increase is more pronounced for polydisperse primary spheres than for monodisperse ones. On the other hand, the dimensionless large scale cutoff parameter decreases with the agglomerate size. This is mathematically explained by the decrease of the $\beta$ with $N_{p}$ since $\xi_{\infty} / R_{g}$ is driven by $\beta$ and $D_{f i}$ for large agglomerates (see Eq.19). It can be seen that the forcing of $D_{f i}$ has a limited impact on these two dimensionless parameters.
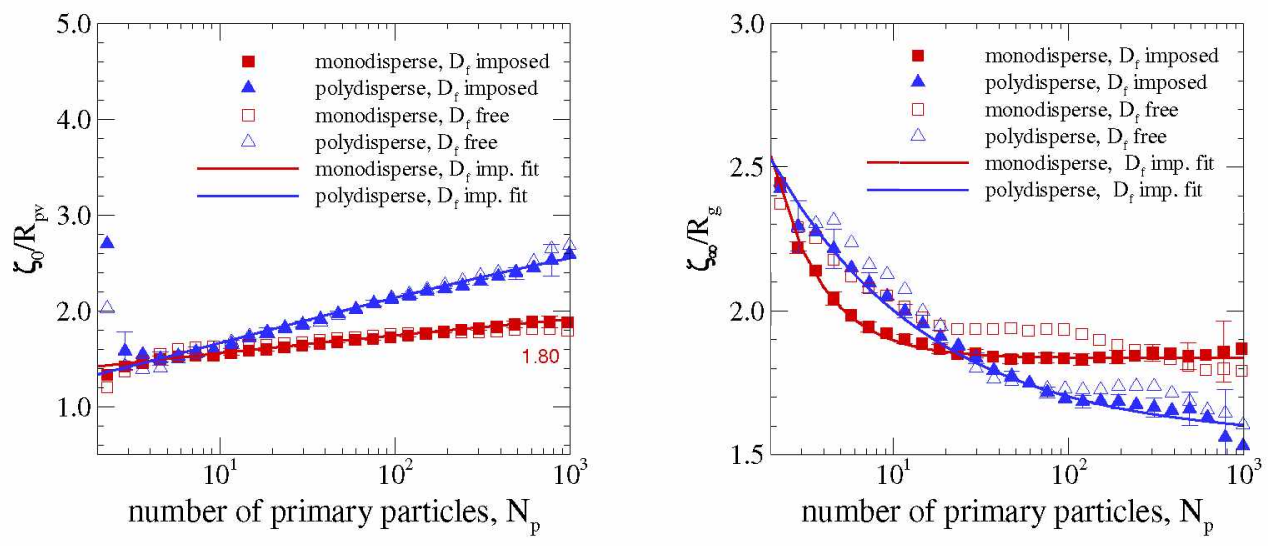

Figure 4: Fitted size parameters of the cutoff functions at small and large scales as a function of the number of primary particles. Error bars correspond to the $95 \%$ confidence intervals.

\subsection{Application of the model to the determination of the fractal prefactor and agglomerates structure factor}

To summarize, independently of the number and the polydispersity of the primary spheres, the agglomerate volume (Eq. 13), radius of gyration (Eq. 15), individual prefactor (Eq. 16) and structure factor (Eq. 20) can be 
expressed as a function of $D_{f i}, \beta, \varphi, \xi_{\infty}, \xi_{0}$ by the following set of equations:

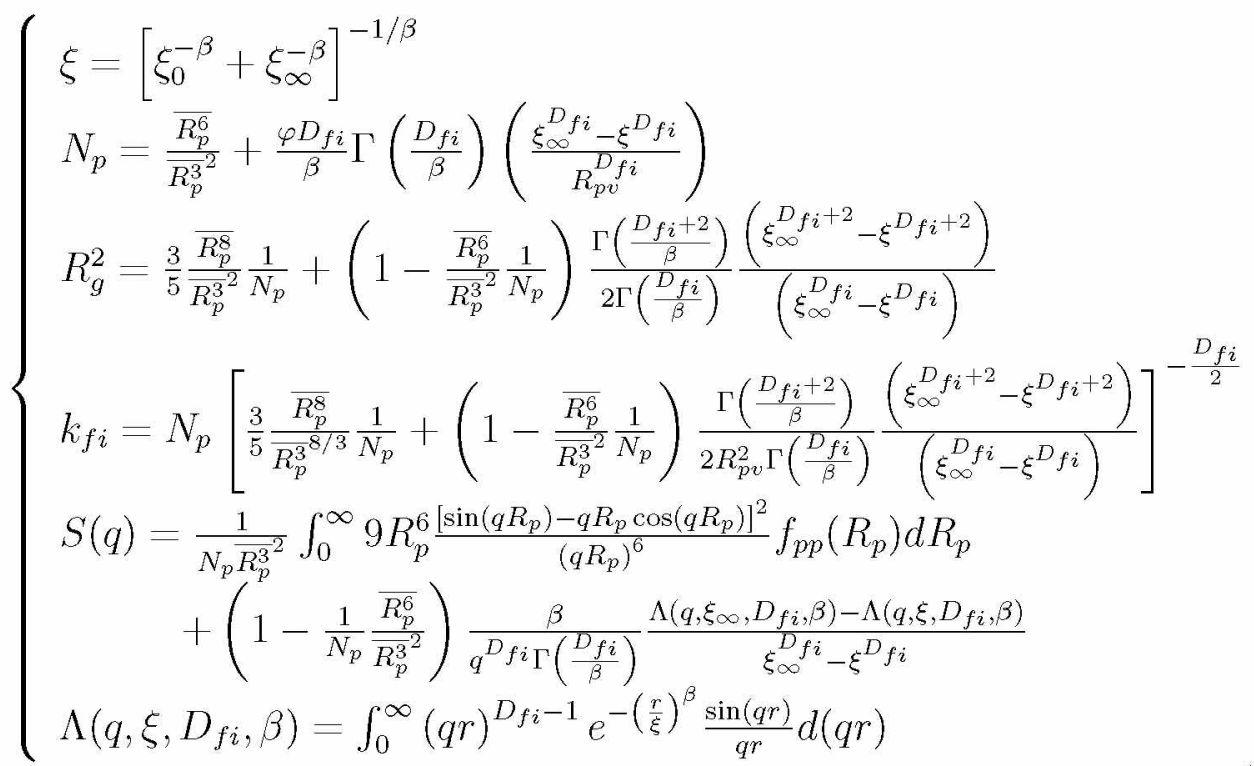

In what follows, in order to illustrate the modelling of the fractal prefactor and structure factor, equations 26 are considered along with the analysis of the parameters dependence on $N_{p}$ based on the fitting process with $D_{f i}$ imposed to 1.64 (plain curves in Figs. 3,4 and corresponding equations reported in Appendix A).

Figure 5 presents in fill symbols the solution of Eq. 26 for the modelling of the individual fractal prefactor by considering $D_{f i}=1.64$. The results show that the individual fractal prefactor never reaches a plateau and a dependence on $N_{p}$ should be considered, in particular for polydisperse primary spheres (red curve). But, even for the monodisperse case for which a asymptotic value seems attainable, $k_{f i}$ is still varying at $N_{p}$ 's recommended by previous studies Ehrl et al. (2009); Lazzari et al. (2016); Isella and Drossinos (2010). The open symbols in Fig. 5 correspond to the application of the asymptotic derivation proposed by Heinson et al. (2012) (Eq. 7) by considering the dependence of $\beta$ and $\varphi$ on $N_{p}$ as reported in Fig. 3 and in Appendix A. There is clearly a very good agreement with the present model for large agglomerates. The difference between both approaches is an indication of the influence played by the cutoff at small scales and by taking into account, the primary spheres self-intersection $A_{p p}$ that represents the novelty of the present study. 
Surprisingly, it appears that approximately 70 and 40 primary spheres respectively are necessary in the monodisperse and polydisperse case to make the influence of the small scales negligible. This shows the importance of taking into account small scale effects, in particular as most of particles have typical sizes in this range, such as soot particles that have an average number of primary spheres close to 100 Köylü and Faeth (1992); Yon et al. (2018); Sorensen et al. (1992b).

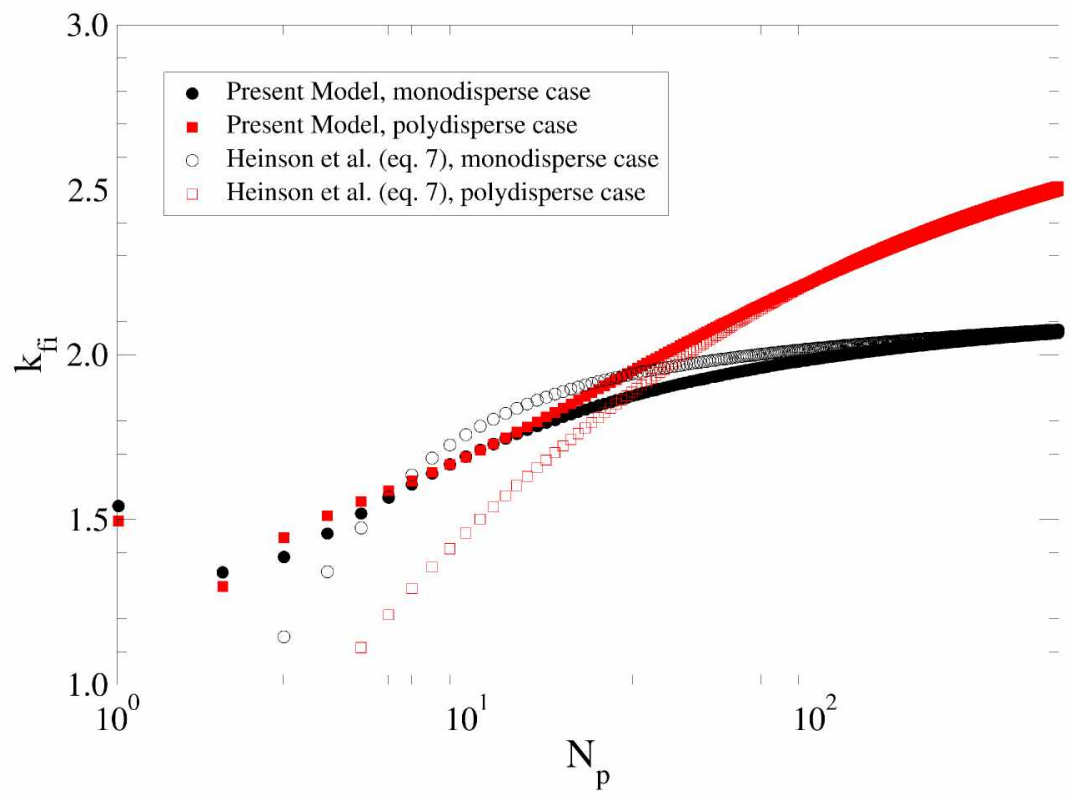

Figure 5: Application of the model to the determination of the individual fractal prefactor $k_{f i}$ depending on the number of primary spheres.

Fig. 5 clearly reports larger $k_{f i}$ for large agglomerates made of polydisperse spheres compared to the monodisperse ones. This is related to the previously observed larger packing factor, as depicted in the left half of Fig. 3. As an illustration, the ratio between $k_{f i}$ in polydisperse and monodisperse cases is found to be 1.11 for $N_{p}=100$, which is in good agreement with the ratio of $k_{f p}$ that is found to be 1.07. But, in addition to varying with $N_{p}, k_{f i}$ is found to be much larger than $k_{f p}$. This can also explain why the population based fractal dimension $D_{f p}=1.77$ is found to be larger than 
$D_{f i}=1.64$.

These findings are confirmed by Fig. 6, which presents the classical population based mass fractal plot for both monodisperse and polydisperse studied agglomerates. The points correspond to each considered individual agglomerate in the present study and the continuous red curves correspond to the present model (with $D_{f i}$ imposed at 1.64 and a variable $k_{f i}$ reported in fig. 5). It can be clearly seen that the model is in good agreement with the points scatter. Also, the fitting of the model's curves by a classical fractal law produces population based fractal parameters (equations in red in Fig. 6) in good agreement with the ones determined from the points scatter and thus with conventional values of $D_{f i}$ and $k_{f i}$. This corroborates the observations made by Wozniak et al. (2012), suggesting that the conventional fractal dimension determined based on the fractal plot is not exactly representative of the individual one expressed by the pair correlation function (or structure factor as explained in the next section). This is explained by the important role played by the cutoff functions making the agglomerate not really fractal but "quasi-fractal".

Figure 7 presents the result of the modelling of the structure factor for different values of $N_{p}$ from 1 to 300 in the monodisperse (upper half) and the polydisperse case (bottom half). The structure factors are multiplied by the square of the agglomerate's volume because the scattered light intensity is proportional to that product. It also enables the curves to be distinguished graphically. The abscissa is the classical dimensionless form of the $q$ parameter by considering the radius of gyration of the agglomerate. The figure illustrates the ability of the proposed model to evaluate the structure factor of agglomerates independently of their size and taking polydispersity into account. Indeed, the model reproduces the Porod's slope (-4) as illustrated for $N_{p}=1$ and corresponding oscillations in the monodisperse case. It is also able to reproduce the Guinier and power-law regimes for $q R_{g} \leq 3$ and $3 \leq q R_{g} \leq 30$ respectively as illustrated by the relatively good agreement with the Dobbins and Megaridis structure factor Dobbins and Megaridis (1991) for fractal agglomerates reported in the dashed green curves. Nevertheless, unlike the Dobbins and Megaridis' structure factor (RDG-FA), the current model enables the transition from power-law regime toward Porod's regime at large $q$, to be determined. This is of interest in particular for the interpretation of SAXS measurements since the range of q covered by X-rays for nanoparticles usually contains this transition regime between power-law and Porod's law. 

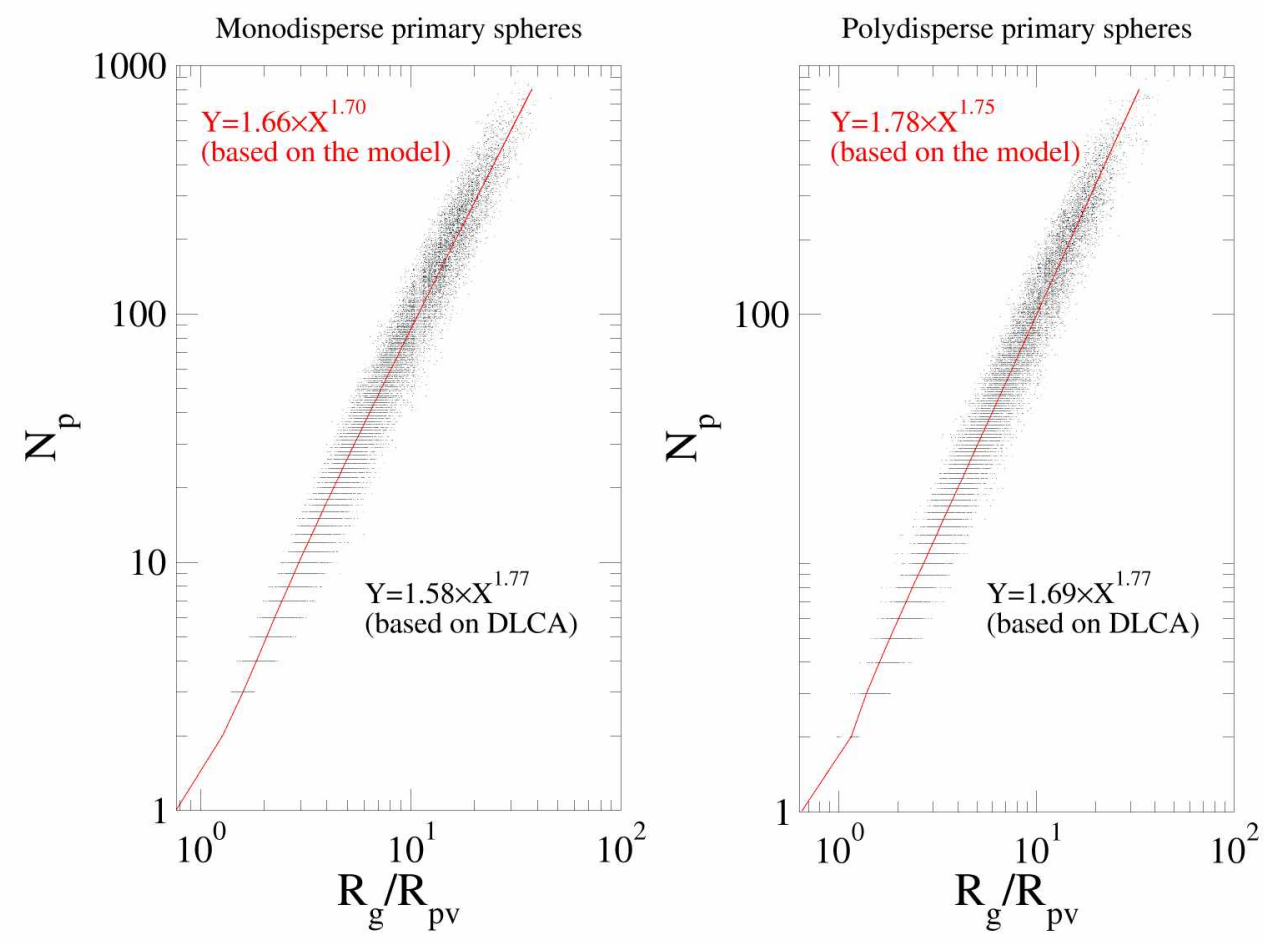

Figure 6: Fractal Plots. Each dot corresponds to a given agglomerate, the continuous red curve corresponds to the proposed modelling.

In addition to the particle size determination, one of the most usual uses of Static Light Scattering with visible light or SAXS with X rays, is the determination of the local slopes in the log-log representation of the structure factor in the power-law and Porod's law regimes, which provide $-D_{f i}$ for large agglomerates and -4 for smooth primary spheres respectively. To discuss this ability, the magnitude of the local slopes based on the proposed model for the polydisperse case and different agglomerate sizes $\left(N_{p}\right)$ is plotted in Fig. 8. The dashed lines represent the expected values for the power-law and Porod's law. The figure illustrates that particular care must be taken when interpreting the local slopes for both discussed regimes. Indeed, even for large agglomerates $\left(N_{p}=300\right.$ in the present case), oscillations can be observed in the range $q R_{g}$ between $3-30$, where RDG-FA predicts a plateau 

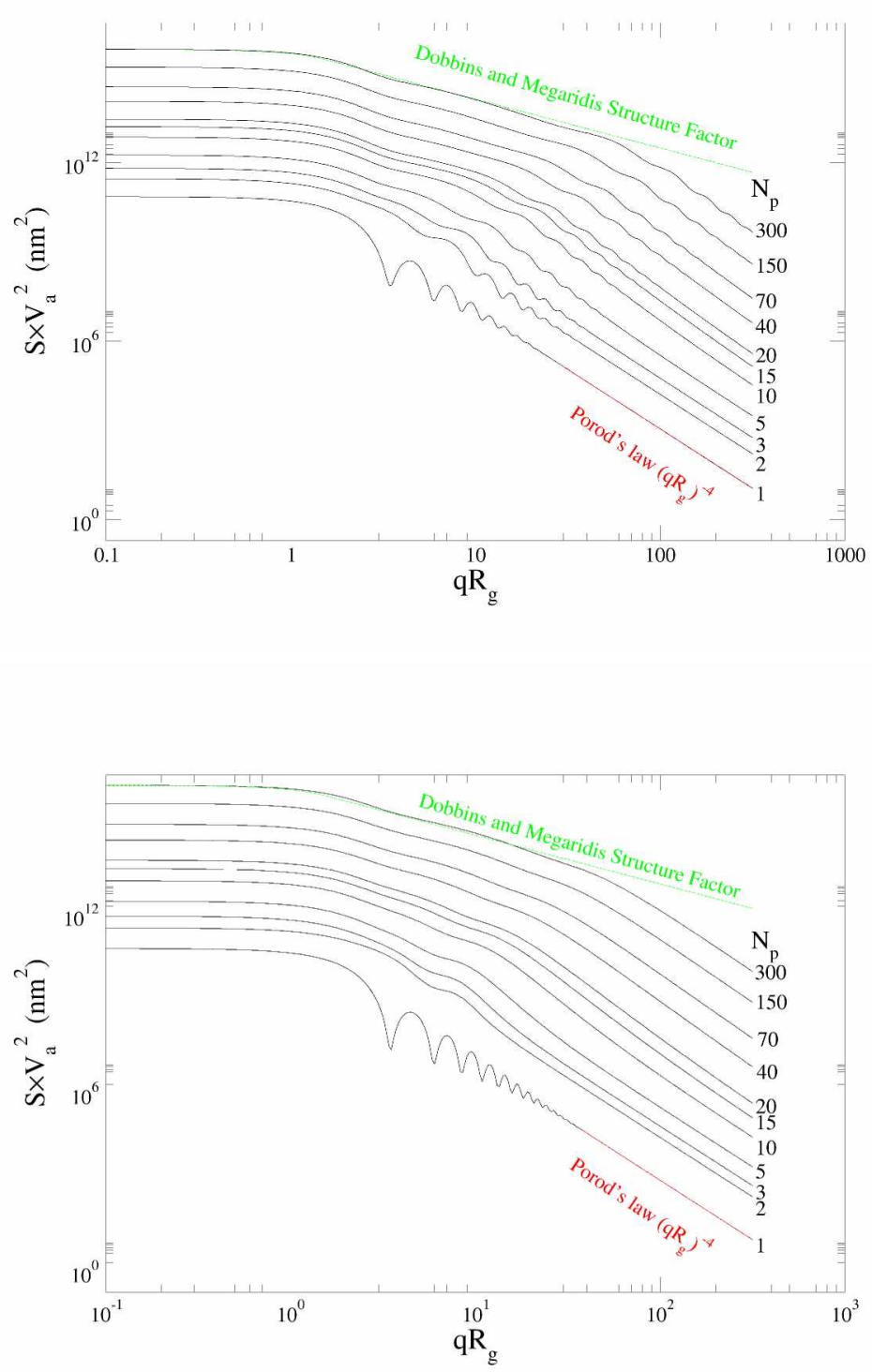

Figure 7: Application of the model to the determination of the structure factor dependence on the number of primary spheres. The upper shows the monodisperse primary spheres case, and bottom half, the polydisperse primary spheres case. 


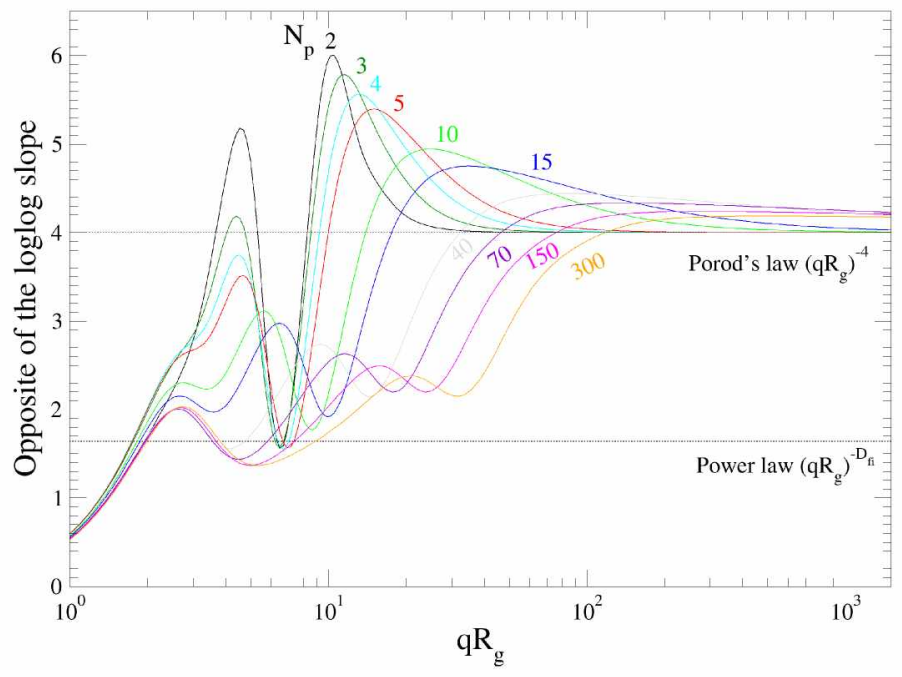

Figure 8: Opposite of the magnitude of the local slope of the modelled structure factor for different number of primary particles in polydisperse case.

equal to the fractal dimension. Consequently, it appears quite illusive to accurately determine the fractal dimension based on a local fitting of the structure factor for quasi-fractal agglomerates. It also appears in Fig. 8 that to arrive at the Porod regime $q R_{g}$ must be larger as the particle size grows. Even if a Porod's law seems established for large $q R_{g}$ in the bottom plot of Figure 7, its fitting can provide different values than 4 even if particles are perfectly smooth spheres in point contact. This is due to the strong influence of the agglomerate structure $A_{\text {agg }}$ up to large $q$. Consequently, it is important to be aware of this effect in order not to interpret this change of slope as an indication of the primary sphere surface roughness as proposed by Beaucage Beaucage (1995); Beaucage et al. (2004), even if this effect cannot be excluded. Note that experimental measurements are smoothed due to the polydisperse nature of the agglomerate population, that is not considered in the present analysis. 


\section{Conclusions}

Experimentally measured nanoparticle agglomerates are usually regarded as fractal-like due to their finite size. In this context, their morphological characterization usually involves the addition of a cut-off function Sorensen (2001); Cai et al. (1995); Sorensen et al. (1992a); Nicolai et al. (1994); Heinson et al. (2012). However, these morphological analyses are also limited at small scales, particularly when approaching the monomer size scale. To the author's knowledge, apart from the work carried out by Lattuada et al. (2003), there are currently no models able to overcome this issue and therefore, the present work is intended to cover this gap. This is achieved by considering the contribution of the primary spheres in the modeling of the pair correlation function as well as by adding a cutoff function at small scales.

This results in a more sophisticated modeling of the pair correlation based on 5 parameters, namely the individual agglomerate's fractal dimension, the packing factor, the stretching exponent and two characteristic size parameters representative of small and large scales plus a parameter describing the primary sphere size distribution. In the limit of large agglomerates, the proposed method gives results in good agreement with previous work Ehrl et al. (2009); Lazzari et al. (2016).

The dependence of the model parameters on agglomerate size has been investigated by computing the pair correlation functions of numerically generated DLCA agglomerates. Analytical expressions for the individual fractal prefactor and structure factor are also derived from the proposed model.

One important result is that the classical fractal law based on a population of agglomerates is not suitable for determining the individual fractal dimension, due to the limitations in fractal geometry induced by both small and large scales. Indeed, the fractal prefactor is shown to strongly evolve with agglomerate size, thus resulting in an overestimation of the effective population based fractal dimension (classically 1.77 for DLCA agglomerates) compared to the individual one (found to be 1.64 in the present study). Consequently, the agglomerates have to be extremely large (constituted of a very large number of primary spheres, more than 100) to avoid the bias induced by the finite size of the particle. Therefore, the present work highlights the bias induced by small scales that is shown to be particularly important when considering the polydispersity of primary spheres. Furthermore, it is found that the local porosity (packing factor) also evolves with particle size.

The present work also enables the unified modelling of the structure fac- 
tor for quasi-fractal agglomerates to be carried out, regardless the number of primary spheres and taking polydispersity into account. This work therefore extends the field of validity of the RDG-FA model Sorensen (2001); Dobbins and Megaridis (1991) and provides a theoretical basis for the scaling approaches as proposed by Beaucage Beaucage (1995). Supplementing the investigation by Lattuada et al. (2003), this work enables the modelling to be carried out of the transition between Guinier and Power-law regimes as well as between Power-law and Porod's law regimes. This approach is thus a promising and complementary tool to the RDG-FA and Beaucage models for the interpretation of visible or X-ray light scattering experiments for agglomerates constituted of a limited number of primary spheres.

The aim of the present model is for the analysis of experimental TEM/SEM images of nanoparticles agglomerates, especially at early stages of particle formation where agglomerates consist of a few number of monomers Bescond et al. (2014); Ouf et al. (2019). Indeed, correlations for agglomerates projected areas, collision radius, etc. may be adapted to small agglomerates based on the current model Brasil et al. (1999); Thajudeen et al. (2012). Also, the numerical simulations of nanoparticle coagulation based on the population balance equation, lack models for agglomerates morphology ranging from one monomer to large agglomerates Kostoglou and Konstandopoulos (2001). The same problem is found for tunable algorithms where the uncertainty of selecting constant fractal parameters during the agglomeration of particles still exists Morán et al. (2019); Singh and Tsotsas (2020). The use of the present model in tunable codes is promising in order to generate more realistic morphologies, closer to those produced by Diffusion Limited particle-Cluster Agglomeration Meakin (1999); Eggersdorfer and Pratsinis (2012). Future developments of the proposed model should take into account, primary sphere overlapping, more realistic morphologies and aggregates size polydispersity.

\section{Acknowledgments}

This work was financed by ANR ASTORIA (N ANR-18-CE05-0015) and the Region of Normandy (project RIN Gaspropres). We also thank the CRIANN numerical resources supported by the Normandy region. 
${ }_{408}$ Appendix A. Fits of the pair correlation function parameters

409 Appendix A.1. Monodisperse monomers

Based on the imposed fractal dimension $D_{f i}=1.64$

$$
\begin{gathered}
\beta=3.203+\exp \left(-1.476\left(\log \left(N_{p}\right)-1.592\right)\right) \\
\frac{\xi_{0}}{R_{p v}}=\frac{2.599}{1+\exp \left(-0.135\left(\log \left(N_{p}\right)+0.687\right)\right)} \\
\frac{\xi_{\infty}}{R_{g}}=1.836+\exp \left(-1.510\left(\log \left(N_{p}\right)-0.457\right)\right) \\
\phi=1.080-\exp \left(-0.334\left(\log \left(N_{p}\right)+0.116\right)\right) \\
\Gamma\left(D_{f} / \beta\right)=1.36+\frac{1.54}{\ln N_{p}} \\
\frac{\Gamma\left(\frac{D_{f i}+2}{\beta}\right)}{2 \Gamma\left(\frac{D_{f i}}{\beta}\right)}=0.31-0.19 \frac{\ln N_{p}}{N_{p}}
\end{gathered}
$$

${ }_{410}$ Appendix A.2. Polydisperse monomers

Based on the imposed fractal dimension $D_{f i}=1.64$ :

$$
\begin{gathered}
\beta=2.496+\exp \left(-0.739\left(\log \left(N_{p}\right)-2.778\right)\right) \\
\frac{\xi_{0}}{R_{p v}}=\frac{3.448}{1+\exp \left(-0.242\left(\log \left(N_{p}\right)-2.587\right)\right)} \\
\frac{\xi_{\infty}}{R_{g}}=1.554+\exp \left(-0.481\left(\log \left(N_{p}\right)-0.632\right)\right) \\
\phi=1.840-\exp \left(-0.220\left(\log \left(N_{p}\right)-3.123\right)\right) \\
\Gamma\left(D_{f} / \beta\right)=0.93+\frac{2.26}{\ln N_{p}} \\
\frac{\Gamma\left(\frac{D_{f i}+2}{\beta}\right)}{2 \Gamma\left(\frac{D_{f i}}{\beta}\right)}=0.37-0.39 \frac{\ln N_{p}}{N_{p}}
\end{gathered}
$$


${ }_{411}$ Appendix B. The evolution of the average coordination number

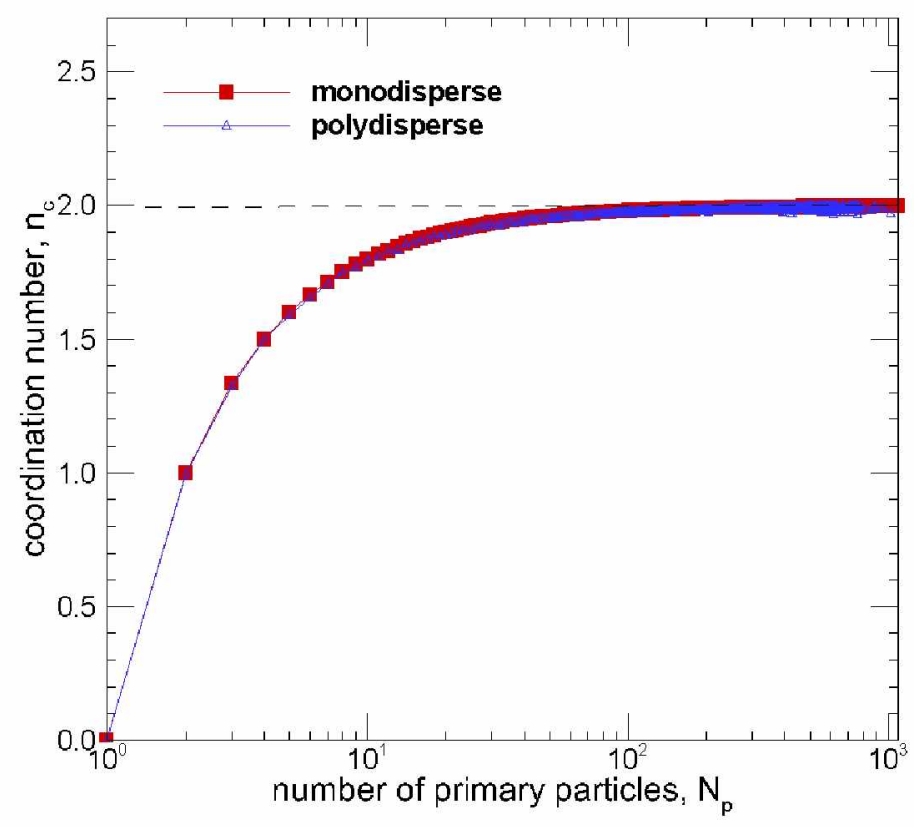

Figure B.9: Average coordination number as a function of the number of primary particles. 


\section{References}

Altenhoff, M., Aßmann, S., Perlitz, J.F., Huber, F.J., Will, S., 2019. Soot aggregate sizing in an extended premixed flame by high-resolution twodimensional multi-angle light scattering (2d-mals). Applied Physics B 125, 176. doi:https://doi.org/10.1007/s00340-019-7282-0.

Altenhoff, M., Aßmann, S., Teige, C., Huber, F.J., Will, S., 2020. An optimized evaluation strategy for a comprehensive morphological soot nanoparticle aggregate characterization by electron microscopy. Journal of Aerosol Science 139, 105470.

Beaucage, G., 1995. Approximations leading to a unified exponential/powerlaw approach to small-angle scattering. Journal of Applied Crystallography 28, 717-728. doi:https://doi.org/10.1107/S0021889895005292.

Beaucage, G., Kammler, H.K., Mueller, R., Strobel, R., Agashe, N., Pratsinis, S.E., Narayanan, T., 2004. Probing the dynamics of nanoparticle growth in a flame using synchrotron radiation. Nature Materials 3, 370. doi:https://doi.org/10.1038/nmat1135.

Bescond, A., Yon, J., Ouf, F., Ferry, D., Delhaye, D., Gaffié, D., Coppalle, A., Rozé, C., 2014. Automated determination of aggregate primary particle size distribution by tem image analysis: application to soot. Aerosol Science and Technology 48, 831-841. doi:https://doi.org/10. $1080 / 02786826.2014 .932896$.

Betrancourt, C., Liu, F., Desgroux, P., Mercier, X., Faccinetto, A., Salamanca, M., Ruwe, L., Kohse-Höinghaus, K., Emmrich, D., Beyer, A., et al., 2017. Investigation of the size of the incandescent incipient soot particles in premixed sooting and nucleation flames of n-butane using lii, him, and 1 nm-smps. Aerosol Science and Technology 51, 916-935. doi:https://doi .org/10.1080/02786826.2017.1325440.

Bladh, H., Johnsson, J., Rissler, J., Abdulhamid, H., Olofsson, N.E., Sanati, M., Pagels, J., Bengtsson, P.E., 2011. Influence of soot particle aggregation on time-resolved laser-induced incandescence signals. Applied Physics B 104, 331-341. doi:https://doi .org/10.1007/s00340-011-4470-y.

Bouvier, M., Yon, J., Lefevre, G., Grisch, F., 2019. A novel approach for insitu soot size distribution measurement based on spectrally resolved light 
scattering. Journal of Quantitative Spectroscopy and Radiative Transfer 225, 58-68. doi:https://doi.org/10.1016/j.jqsrt.2018.12.018.

Brasil, A., Farias, T., Carvalho, M., 1999. A recipe for image characterization of fractal-like aggregates. Journal of Aerosol Science 30, 1379. doi:https : //doi.org/10.1016/S0021-8502(99)00026-9.

Bushell, G., Amal, R., 1998. Fractal aggregates of polydisperse particles. Journal of colloid and interface science 205, 459-469. doi:10.1006/jcis. 1998.5667.

Bushell, G., Amal, R., 2000. Measurement of fractal aggregates of polydisperse particles using small-angle light scattering. Journal of colloid and interface science 221, 186-194. doi:https://doi.org/10.1006/jcis. 1999. 6532.

Bushell, G., Yan, Y., Woodfield, D., Raper, J., Amal, R., 2002. On techniques for the measurement of the mass fractal dimension of aggregates. Advances in Colloid and Interface Science 95, 1-50. doi:https ://doi .org/10.1016/ S0001-8686(00)00078-6.

Cai, J., Lu, N., Sorensen, C.M., 1995. Analysis of fractal cluster morphology parameters: structural coefficient and density autocorrelation function cutoff. Journal of Colloid and Interface Science 171, 470-473. doi:https://doi.org/10.1006/jcis.1995.1204.

Cortés, D., Morán, J., Liu, F., Escudero, F., Consalvi, J.L., Fuentes, A., 2018. Effect of fuels and oxygen indices on the morphology of soot generated in laminar coflow diffusion flames. Energy \& fuels 32, 11802-11813. doi:https://doi .org/10.1021/acs . energyfuels.8b01301.

Debye, P., Anderson Jr, H., Brumberger, H., 1957. Scattering by an inhomogeneous solid. ii. the correlation function and its application. Journal of applied Physics 28, 679-683.

Dobbins, R., Megaridis, C., 1991. Absorption and scattering of light by polydisperse aggregates. Appl. Opt. 30, 4747-4754. doi:https://doi. org/10.1364/A0.30.004747. 
Eggersdorfer, M.L., Pratsinis, S.E., 2012. The structure of agglomerates consisting of polydisperse particles. Aerosol science and technology 46, 347-353. doi:https://doi .org/10.1080/02786826.2011.631956.

Ehrl, L., Soos, M., Lattuada, M., 2009. Generation and geometrical analysis of dense clusters with variable fractal dimension. The Journal of Physical Chemistry B 113, 10587-10599. doi:https://doi.org/10.1021/ jp903557m.

Filippov, A., Zurita, M., Rosner, D., 2000. Fractal-like aggregates: relation between morphology and physical properties. Journal of colloid and interface science 229, 261-273. doi:10.1006/jcis.2000.7027.

Gigone, B., Karataş, A.E., Gülder, Ö.L., 2019. Soot aggregate morphology in coflow laminar ethylene diffusion flames at elevated pressures. Proceedings of the Combustion Institute 37, 841-848. doi:https://doi.org/10.1016/ j.proci.2018.06.103.

Gmachowski, L., 2002. Calculation of the fractal dimension of aggregates. Colloids and Surfaces A: Physicochemical and Engineering Aspects 211, 197-203. doi:https://doi org/10.1016/S0927-7757 (02)00278-9.

Heinson, W.R., Sorensen, C.M., Chakrabarti, A., 2012. A three parameter description of the structure of diffusion limited cluster fractal aggregates. Journal of colloid and interface science 375, 65-69. doi:https://doi .org/ $10.1016 / j \cdot j$ cis.2012.01.062.

Isella, L., Drossinos, Y., 2010. Langevin agglomeration of nanoparticles interacting via a central potential. Physical Review E 82, 011404. doi:https: //doi.org/10.1103/PhysRevE.82.011404.

Jullien, R., 1992. The application of fractals to colloidal aggregation. Croatica Chemica Acta 65, 215-235.

Kempema, N.J., Long, M.B., 2016. Combined optical and tem investigations for a detailed characterization of soot aggregate properties in a laminar coflow diffusion flame. Combustion and Flame 164, 373-385. doi:https: //doi.org/10.1016/j. combustflame.2015.12.001.

Kholghy, M., Saffaripour, M., Yip, C., Thomson, M.J., 2013. The evolution of soot morphology in a laminar coflow diffusion flame of a surrogate for 
jet a-1. Combustion and Flame 160, 2119-2130. doi:https://doi.org/ $10.1016 / j$. combustflame.2013.04.008.

Kostoglou, M., Konstandopoulos, A.G., 2001. Evolution of aggregate size and fractal dimension during brownian coagulation. Journal of Aerosol Science 32, 1399-1420. doi:https://doi .org/10.1016/S0021-8502(01) 00056-8.

Köylü, Ü.Ö., Faeth, G., Farias, T.L., Carvalho, M.d.G., 1995. Fractal and projected structure properties of soot aggregates. Combustion and Flame 100, 621-633. doi:https://doi.org/10.1016/0010-2180(94)00147-K.

Köylü, Ü.Ö., McEnally, C.S., Rosner, D.E., Pfefferle, L.D., 1997. Simultaneous measurements of soot volume fraction and particle size/microstructure in flames using a thermophoretic sampling technique. Combustion and Flame 110, 494-507. doi:https://doi.org/10.1016/S0010-2180(97) 00089-8.

Köylü, , Faeth, G.M., 1992. Structure of overfire soot in buoyant turbulent diffusion flames at long residence times. Combustion and Flame 89, 140156. doi:https://doi .org/10.1016/0010-2180(92)90024-J.

Lapuerta, M., Martos, F.J., Martín-González, G., 2010. Geometrical determination of the lacunarity of agglomerates with integer fractal dimension. Journal of colloid and interface science 346, 23-31. doi:https: //doi.org/10.1016/j.jcis.2010.02.016.

Lattuada, M., Ehrl, L., 2009. Scattering properties of dense clusters of colloidal nanoparticles. The Journal of Physical Chemistry B 113, 5938-5950. doi:10.1021/jp810976z.

Lattuada, M., Wu, H., Morbidelli, M., 2003. A simple model for the structure of fractal aggregates. Journal of Colloid and Interface Science 268, 106-120. doi:https://doi.org/10.1016/j.jcis.2003.07.027.

Lazzari, S., Nicoud, L., Jaquet, B., Lattuada, M., Morbidelli, M., 2016. Fractal-like structures in colloid science. Advances in colloid and interface science 235, 1-13. doi:https://doi.org/10.1016/j.cis.2016.05.002.

Lee, K.O., Megaridis, C.M., Zelepouga, S., Saveliev, A.V., Kennedy, L.A., Charon, O., Ammouri, F., 2000. Soot formation effects of oxygen concentration in the oxidizer stream of laminar coannular nonpremixed 
methane/air flames. Combustion and Flame 121, 323-333. doi:https: //doi.org/10.1016/S0010-2180(99)00131-5.

Lefevre, G., Yon, J., Bouvier, M., Liu, F., Coppalle, A., 2019. Impact of organic coating on soot angular and spectral scattering properties. Environmental science \& technology 53, 6383-6391. doi:https://doi.org/ 10.1021 /acs .est. 8b05482.

Lin, M., Klein, R., Lindsay, H., Weitz, D.A., Ball, R.C., Meakin, P., 1990. The structure of fractal colloidal aggregates of finite extent. Journal of colloid and interface science 137, 263-280. doi:https://doi.org/10.1016/ 0021-9797(90)90061-R.

Meakin, P., 1991. Fractal aggregates in geophysics. Reviews of Geophysics 29, 317-354. doi:https://doi.org/10.1029/91RG00688.

Meakin, P., 1999. A historical introduction to computer models for fractal aggregates. Journal of Sol-Gel Science and Technology 15, 97-117. doi:https://doi.org/10.1023/A:1008731904082.

Morán, J., Fuentes, A., Liu, F., Yon, J., 2019. Fracval: An improved tunable algorithm of cluster-cluster aggregation for generation of fractal structures formed by polydisperse primary particles. Computer Physics Communications 239, 225-237. doi:10.1016/j.cpc.2019.01.015.

Morán, J., Yon, J., Poux, A., 2020. Monte carlo aggregation code (MCAC) part 1: Fundamentals. Journal of Colloid and Interface Science 569, $184-$ 194. doi:https://doi.org/10.1016/j.jcis.2020.02.039.

Morán, J., Yon, J., Poux, A., Corbin, F., Ouf, F.X., Siméon, A., 2020. Monte carlo aggregation code (MCAC) part 2: Application to soot agglomeration, highlighting the importance of primary particles. Journal of Colloid and Interface Science 575, 274-285. URL: http://www. sciencedirect. com/science/article/pii/S0021979720305336, doi:https://doi.org/ 10.1016/j.jcis.2020.04.085.

Nicolai, T., Durand, D., Gimel, J.C., 1994. Static structure factor of dilute solutions of polydisperse fractal aggregates. Physical Review B 50, 16357. doi:https://doi.org/10.1103/PhysRevB.50.16357. 
Oltmann, H., Reimann, J., Will, S., 2010. Wide-angle light scattering (wals) for soot aggregate characterization. Combustion and Flame 157, 516-522. doi:https://doi .org/10.1016/j . combustflame. 2009.10.011.

Ouf, F.X., Bourrous, S., Vallières, C., Yon, J., Lintis, L., 2019. Specific surface area of combustion emitted particles: Impact of primary particle diameter and organic content. Journal of Aerosol Science 137, 105436. doi:https://doi.org/10.1016/j. jaerosci.2019.105436.

Rottereau, M., Gimel, J.C., Nicolai, T., Durand, D., 2004. Monte carlo simulation of particle aggregation and gelation: Ii. pair correlation function and structure factor. The European Physical Journal E 15, 141-148. doi:https://doi.org/10.1140/epje/i2004-10045-9.

Singh, A.K., Tsotsas, E., 2020. A tunable aggregation model incorporated in monte carlo simulations of spray fluidized bed agglomeration. Powder Technology 364, 417-428. doi:https://doi.org/10.1016/j . powtec. 2020.02 .016 .

Sorensen, C., 2001. Light scattering by fractal aggregates: a review. Aerosol Science \& Technology 35, 648-687. doi:https://doi.org/10. $1080 / 02786820117868$.

Sorensen, C., Cai, J., Lu, N., 1992a. Test of static structure factors for describing light scattering from fractal soot aggregates. Langmuir 8, 20642069. doi:https://doi.org/10.1021/la00044a029.

Sorensen, C., Lu, N., Cai, J., 1995. Fractal cluster size distribution measurement using static light scattering. Journal of Colloid and Interface Science 174, 456-460. doi:https://doi.org/10.1006/jcis.1995.1413.

Sorensen, C.M., Cai, J., Lu, N., 1992b. Light-scattering measurements of monomer size, monomers per aggregate, and fractal dimension for soot aggregates in flames. Applied Optics 31, 6547-6557. doi:https://doi. org/10.1364/AO.31.006547.

Sorensen, C.M., Roberts, G.C., 1997. The prefactor of fractal aggregates. Journal of colloid and interface science 186, 447-452. doi:https://doi. org/10.1006/jcis.1996.4664. 
Tang, Q., Cai, R., You, X., Jiang, J., 2017. Nascent soot particle size distributions down to $1 \mathrm{~nm}$ from a laminar premixed burner-stabilized stagnation ethylene flame. Proceedings of the Combustion Institute 36, 993-1000. doi:https://doi.org/10.1016/j.proci.2016.08.085.

Thajudeen, T., Gopalakrishnan, R., Hogan Jr, C.J., 2012. The collision rate of nonspherical particles and aggregates for all diffusive knudsen numbers. Aerosol Science and Technology 46, 1174-1186. doi:https://doi .org/10. 1080/02786826.2012.701353.

Wentzel, M., Gorzawski, H., Naumann, K.H., Saathoff, H., Weinbruch, S., 2003. Transmission electron microscopical and aerosol dynamical characterization of soot aerosols. Journal of aerosol science 34, 1347-1370. doi:https://doi org/10.1016/S0021-8502(03)00360-4.

Wozniak, M., Onofri, F., Barbosa, S., Yon, J., Mroczka, J., 2012. Comparison of methods to derive morphological parameters of multi-fractal samples of particle aggregates from tem images. Journal of Aerosol Science 47, 12-26. doi:https://doi.org/10.1016/j. jaerosci.2011.12.008.

Wu, M.K., Friedlander, S.K., 1993. Note on the power law equation for fractal-like aerosol agglomerates. Journal of colloid and interface science 159, 246-248. doi:https://doi.org/10.1006/jcis.1993.1319.

Yon, J., Ouf, F.X., Hebert, D., Mitchell, J.B., Teuscher, N., Le Garrec, J.L., Bescond, A., Baumann, W., Ourdani, D., Bizien, T., et al., 2018. Investigation of soot oxidation by coupling lii, saxs and scattering measurements. Combustion and Flame 190, 441-453. doi:https: //doi.org/10.1016/j. combustflame.2017.12.014.

Zhao, B., Uchikawa, K., Wang, H., 2007. A comparative study of nanoparticles in premixed flames by scanning mobility particle sizer, small angle neutron scattering, and transmission electron microscopy. Proceedings of the Combustion Institute 31, 851-860. doi:https://doi .org/10.1016/j . proci.2006.08.064. 